\title{
Using multiple correspondence analysis to identify behaviour patterns associated with overweight and obesity in Vanuatu adults
}

Andrew van Horn ${ }^{1,2}$, Charles A Weitz ${ }^{1, *}$, Kathryn M Olszowy ${ }^{3}$, Kelsey N Dancause ${ }^{4}$, Cheng Sun 5,6 , Alysa Pomer ${ }^{7}$, Harold Silverman ${ }^{8}$, Gwang Lee 5,6 , Leonard Tarivonda ${ }^{9}$, Chim W Chan ${ }^{10}$, Akira Kaneko ${ }^{10,11,12}$, J Koji Lum ${ }^{5,6,13}$ and Ralph M Garruto $5,13,14$

'Department of Anthropology, Temple University, 214 Gladfelter Hall, 1801 N. Broad Street, Philadelphia, PA 19122, USA: ${ }^{2}$ Center for the Advanced Study of Human Paleobiology, The George Washington University, Washington, DC, USA: ${ }^{3}$ Department of Criminology, Anthropology, and Sociology, Cleveland State University, Cleveland, OH, USA: ${ }^{4}$ Department of Physical Activity Sciences, Université du Québec à Montréal (UQAM), Montréal, QC, Canada: ${ }^{5}$ Department of Anthropology, SUNY Binghamton, Binghamton, NY, USA: ${ }^{6}$ Laboratory of Evolutionary Anthropology and Health, SUNY Binghamton, Binghamton, NY, USA: ${ }^{7}$ Department of Chronic Disease Epidemiology, Yale School of Public Health, New Haven, CT, USA: ${ }^{8}$ Center for Bioelectronic Medicine and Biomedical Science, The Feinstein Institute for Medical Research, Manhasset, NY, USA: ${ }^{9}$ Ministry of Health, Port Vila, Republic of Vanuatu: ${ }^{10}$ Island Malaria Group, Department of Microbiology, Tumor and Cell Biology, Karolinska Institutet, Stockholm, Sweden: ${ }^{11}$ Department of Parasitology and Research Center for Infectious Disease Sciences, Osaka City University Graduate School of Medicine, Osaka, Japan: ${ }^{12}$ Institute of Tropical Medicine, Nagasaki University, Nagasaki, Japan: ${ }^{13}$ Department of Biological Sciences, SUNY Binghamton, Binghamton, NY, USA:

${ }^{14}$ Laboratory of Biomedical Anthropology and Neurosciences, SUNY Binghamton, Binghamton, NY, USA

Submitted 17 July 2018: Final revision received 20 December 2018: Accepted 14 January 2019: First published online 8 March 2019

\begin{abstract}
Objective: The present study evaluates the use of multiple correspondence analysis (MCA), a type of exploratory factor analysis designed to reduce the dimensionality of large categorical data sets, in identifying behaviours associated with measures of overweight/obesity in Vanuatu, a rapidly modernizing Pacific Island country.

Design: Starting with seventy-three true/false questions regarding a variety of behaviours, MCA identified twelve most significantly associated with modernization status and transformed the aggregate binary responses of participants to these twelve questions into a linear scale. Using this scale, individuals were separated into three modernization groups (tertiles) among which measures of body fat were compared and OR for overweight/obesity were computed.

Setting: Vanuatu.

Participants: Ni-Vanuatu adults ( $n$ 810) aged $20-85$ years.

Results: Among individuals in the tertile characterized by positive responses to most of or all the twelve modernization questions, weight and measures of body fat and the likelihood that measures of body fat were above the US 75th percentile were significantly greater compared with individuals in the tertiles characterized by mostly or partly negative responses.

Conclusions: The study indicates that MCA can be used to identify individuals or groups at risk for overweight/obesity, based on answers to simply-put questions. MCA therefore may be useful in areas where obtaining detailed information about modernization status is constrained by time, money or manpower.
\end{abstract}

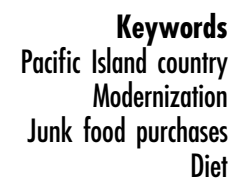

Understanding the social and economic conditions that have resulted in the increased prevalence of overweight/ obesity and associated non-communicable diseases in rapidly modernizing populations of the Southwest Pacific has been a long-term interest of human biologists ${ }^{(1,2)}$. A number of different strategies have been used to identify the causes of overweight/obesity and its health consequences among modernizing South Pacific populations. A common approach has been to compare subgroups of a larger population living in recognizably different economic or social circumstances. This includes comparisons of rural $v$. urban residence ${ }^{(3)}$, comparing different social 
groups living under modern $v$. traditional conditions ${ }^{(4)}$, individuals living on islands that differ in their economic development $^{(5-7)}$ or evaluating the consequences of migration to a more modernized society ${ }^{(8,9)}$. Even though these studies have generally supported the hypothesis that behavioural changes in response to economic development are associated with increases in the prevalence of overweight/obesity, they often do not control for withingroup variability. Thus, presumptions made about different groups (e.g. urban groups are more modern than rural groups) may not necessarily apply to all members. Furthermore, the choice of markers such as income, household composition and occupation may have different sociocultural meanings or be associated with different health-related concerns among individuals (or subgroups) at different points in the traditional-to-modern transition $^{(10,11)}$. Since practices associated with modernity vary widely among different island, sex and age groups in developing Pacific nations ${ }^{(12)}$, traditionally used indices of modernization do not always follow an expected gradient $^{(13)}$.

Recent population studies have used a variety of sophisticated regression and risk factor models to identify within-group, individual behavioural characteristics that are most likely to be associated with overweight/obesity or non-communicable diseases ${ }^{(5,14,15)}$. In addition, a pattern of dietary items that cluster together to explain the variance in obesity and other metabolic syndrome characteristics has been identified using principal component analysis $^{(16)}$. An analogous approach is to use multiple correspondence analysis (MCA). MCA identifies composite dimensions in large categorical data sets in a manner analogous to the way principal component analysis is used to identify latent variables in continuous data ${ }^{(17)}$. Each dimension generated by MCA has a greater potential explanatory capacity than any single variable in the data set and can be represented as a continuous numerical scale that permits direct linear comparisons with any biometric characteristic. This scale can be the foundation of regression analysis or can be sub-divided to produce status categories for the analysis of overweight/obesity $^{(18,19)}$ and other health concerns ${ }^{(20-23)}$.

As in many developing Southwest Pacific island nations, the prevalence of obesity has increased substantially among men and women in Vanuatu over the past 50 years $^{(24,25)}$. However, understanding the reasons behind this increase is complicated by differences in the level of development among Vanuatu's sixty-five inhabited islands ${ }^{(26)}$ and by individual differences in participation in the cash economy within each island. This situation offers an excellent opportunity to apply MCA to identify a set of specific behaviours that collectively are associated with significant differences in individual anthropometric measures of overweight/obesity across all islands. Using MCA in this way takes into consideration both within- and between-island diversity, and makes it possible to develop a more nuanced understanding of the relationship between modernization/development status and overweight/obesity.

\section{Methods}

\section{Samples}

In 2011, the residents of fourteen villages on five Vanuatu islands (Efate, Nguna, Aneityum, Ambae and Futuna) participated in a comprehensive malarial survey, the goal of which was to contact and assess the malarial status of all inhabitants. These islands were selected based on an appraisal of their level of development, determined primarily by their electricity infrastructure, the education and occupation of their inhabitants ${ }^{(26)}$, and on the basis of previous research that showed island differences in morphology ${ }^{(14)}$. Efate clearly represents the most developed island in the survey, since it is the site of the capital city, Port Vila. The level of development is lower in Nguna, and declines progressively on Aneityum, Ambae and Futuna ${ }^{(26)}$.

Most of those who participated in the larger survey also agreed to participate in the current study. Unfortunately, direct comparisons of our sample sizes with village-level population figures cannot be made, because data from the census conducted two years before our study are available only for islands as a whole ${ }^{(27)}$. Nevertheless, three of the study islands (Aneityum, Futuna and Nguna) are small enough so that it is possible to approximate the degree of coverage reasonably accurately. On Aneityum, about $80 \%$ of the 430 adults (20 years or older) enumerated in 2009 lived in the three villages we surveyed. Thus, our sample of 214 adults consisted of about $60 \%$ of the total adult residents of those villages. On Futuna Island, about twothirds of the total adult population (201 enumerated in 2009) lived in the three study villages. Our sample of 114 therefore constitutes approximately $80 \%$ of the adult residents of those villages. We also sampled four of the ten villages on Nguna Island (approximately $40 \%$ of the island's inhabitants). Since the total adult population of Nguna was 669 in 2009, our sample of 188 constituted approximately two-thirds of the resident adult population of the study villages. Supplemental Table 1 (see online supplementary material) shows that the proportions of males and females and the distribution of ages in our samples from Aneityum, Futuna and Nguna did not differ significantly from the age and sex distributions enumerated in 2009 on the islands as a whole.

Both Ambae and Efate are much larger islands, with much larger populations. Local population figures for the Area Council regions on Ambae and Efate within which our study took place are available from a 1999 village enumeration $^{(28)}$ and from a mini-census conducted in $2016^{(29)}$. On Ambae, using figures from the 2016 minicensus to estimate the 2011 population is reasonable, 
since the total island population grew by only $2 \%$ between 2009 and 2016. The population of the Ambae villages from which our sample was drawn constituted $37 \cdot 6 \%$ of the total (i.e. including all ages) South Ambae Area Council population in 1999 (364/967). In 2016, 683 adults were enumerated in the Area Council region. Presuming the proportion of the population in the study villages relative to the Area Council region as a whole remained the same (i.e. 37.6\%), there would have been roughly 257 adults living in the study villages in 2016. In that case, our sample of 110 men and women would have constituted about $43 \%$ of the resident adults. The proportions of men and women in our sample of Ambae villages and the age distribution among males did not differ from those of the total South Ambae Area Council population in 2016; but our sample was skewed towards younger aged women. On Efate Island, the study was conducted in a peri-urban area located about $16 \mathrm{~km}$ (10 miles) from Port Vila, the nation's capital. In 2016, 4935 adults were enumerated in the area where the study village was located. However, using the 2016 mini-census to estimate the coverage in this area is problematic both because the island's population increased significantly (+30\%) between 2009 and 2016, and because a large number of retirees lived in the study village. Our best estimate is based on the presumption that the proportion of the total Area Council region population living in the study village in 1999 (25\% from village-level data) remained the same. In this case, the adult population would have been about 1235 in 2016 (or about 865 in 2011, presuming that the population was $30 \%$ less). Thus, our sample of 184 adult men and women would have constituted between about 15 and $21 \%$ of the total adult inhabitants. Supplemental Table 1 shows that our sample includes a larger proportion of men and a larger proportion of older adults compared with the Area Council region as a whole in 2016.

In sum, our sampling was sufficiently large and appropriately stratified on Aneityum, Futuna, Nguna and likely South Ambae to accurately represent the populations of the villages in which the study took place. Using a 95\% CI, the confidence level for the survey was $\pm 4 \%$ on Aneityum, $\pm 6 \%$ on Futuna and Nguna, and $\pm 7 \%$ on Ambae. Our sample of the study village on Efate may have been less representative (producing a confidence level of $\pm 8 \%$ ). However, there is no way of knowing whether our subjective assessment of the large number of retirees in the village may have made our sampling more accurate than might be indicated by comparisons with the larger Area Council region. At the very least, a larger sample of individuals aged 50 years or older on Efate likely increased our total sample of older adults who might have participated in the cash economy.

Permission to conduct this research was received from the Temple University Institutional Review Board (protocol number 13827) and from the Binghamton University Institutional Review Board (protocol number 1578-10) before the commencement of the study, and permission to conduct research in-country was granted by the Vanuatu Ministry of Health. In each village where the study took place, the research protocol and aims were explained to local chiefs and, at a general meeting, to the community at large. Informed consent was obtained from each adult participant. Institutional Review Boards and the Vanuatu Ministry of Health approved this consent procedure.

\section{Measurements}

An extensive behavioural survey was administered to 810 individuals aged 20 years or older (398 men and 412 women) by trained interviewers. This survey provided information on demographics, family and personal history of illness, food purchasing behaviours, activities, and electronics ownership and technology use. Interviews were conducted in 'Bislama', the lingua franca of Vanuatu. The analysis presented here focuses on seventythree questions that were answered by 'yes/no' (see online supplementary material, Supplemental Table 2). The focus on questions with a simple response (rather than questions that asked individuals for a subjective determination of the extent/degree to which they were involved in various behaviours) was done purposefully to determine if MCA could effectively be used to characterize behaviours associated with differences in overweight/ obesity.

Anthropometric measurements were collected following standard guidelines ${ }^{(30)}$. Standing height without shoes was measured using a Seca 214 stadiometer (Seca, Hamburg, Germany). Weight was measured using a Tanita TBF-521 digital scale (Tanita, Arlington Heights, IL, USA) and percentage body fat (\%BF) was calculated using Tanita proprietary formulas. Weight and height were used to calculate BMI $\left(\mathrm{kg} / \mathrm{m}^{2}\right)$. Lange skinfold callipers (Beta Technologies, Cambridge, MD, USA) were used to determine skinfold thicknesses at the triceps, subscapular and supraliliac sites, and the mean of three measures at each site was used for analyses.

\section{Analytical strategy}

MCA was used to analyse individual responses to yes/no questions about easily recognizable behaviours associated with daily life and to identify behavioural categories that were linked to variation in measures of overweight/obesity. MCA is a type of exploratory factor analysis designed to reduce the dimensionality of large categorical data sets that include more than two variables distributed among a large number of individual responses ${ }^{(31)}$. Thus, MCA can suggest unexpected dimensions and relationships in the tradition of exploratory data analysis. As in related methods of factor analysis like principal component analysis, MCA assumes that the variables comprising a data matrix represent a high-dimensional Euclidean space and collapses this $n$-dimensional space by creating new, synthetic 
dimensions. These dimensions are derived from an indicator matrix of the data set, in which all possible responses to the questions are coded as $0 / 1$ binary variables. Each dimension is a vector that is characterized by a correlated set of related variables. The $\chi^{2}$ distance calculated between each of the variables and between each of the individuals is the basis for determination of explained variance. As in principal component analysis, the first dimension accounts for the greatest variance in the participant-plus-variable profile that makes up the data set. Each subsequent dimension describes the greatest proportion of the remaining variance. Further, MCA is used to determine the coordinates of individuals on each synthetic dimension, which are analogous to factor scores for individual cases in a principal component analysis. Thus, MCA permits individuals to be positioned linearly, based on the pattern of shared responses.

For the analysis of the Vanuatu data, we used two successive MCA to generate the indices for further morphological analyses. Our first MCA was exploratory and included a pool of seventy-three 'yes/no' questions converted to Boolean format (true/false) that adult participants answered. MCA was performed on the indicator matrix using the FactoMineR package in $\mathrm{R}$ with default parameters $^{(32)}$. There were no missing data, and no supplementary continuous variables were included. A scree plot of eigenvalues (a measure of inertia, or variance, accounted for by a dimension) identified two primary dimensions (Dimension 1 eigenvalue $=10 \cdot 965$; Dimension 2 eigenvalue $=6 \cdot 715$; Fig. 1(a)). We identified twenty-two questions that were strongly correlated with either the first or the second dimension using $\eta^{2}$ (the squared correlation of the variable to the dimension). A second MCA was performed on these twenty-two questions in order to focus on variables that individually and collectively contributed most significantly to the inertia (explained variance) determined by the initial MCA of all seventy-three

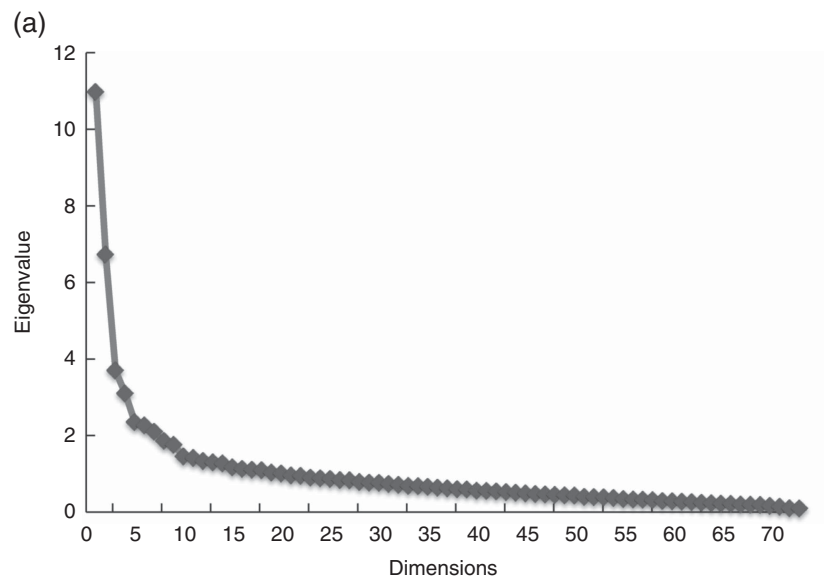

variables (Fig. 1(b)). Figure 2 shows the correlations $\left(\eta^{2}\right)$ between the twenty-two input variables and the two dimensions with the highest eigenvalues identified by the second MCA. The twelve variables showing high $\eta^{2}$ correlations with Dimension 1 explained the greatest amount of the variance $(33.6 \%)$ in this second analysis. These twelve variables (listed in Fig. 2) consisted of responses to questions about foods purchased at stores. Thus, this dimension has been labelled 'food purchasing behaviour'. Factor scores generated by MCA represent the position of each individual on the numerical scale derived from this dimension. These factor scores ranged from those who answered 'yes' to all twelve questions to those who answered 'no' to all twelve questions. Based on their position on this scale, individuals were divided into three tertiles. Individuals whose factors scores were at the upper one-third of the distribution were characterized by answering 'true' to most of or all the questions about food purchases at stores. Individuals whose factor scores were at the lower one-third of the distribution were characterized by answering 'false' to most of or all the questions about food purchases at stores. Those in the middle third answered a mix of 'true' or 'false' to the questions. A number of individuals at the boundary between the middle and upper tertile clustered at the same factor score. These individuals were arbitrarily assigned to the upper tertile. This produces a distribution that has a slightly higher percentage of upper-tertile individuals ( $n_{\text {upper tertile }}$ $276,34 \%)$ compared with the percentage of lower- and middle-tertile individuals ( $n_{\text {lower tertile }} 267,33 \%$; $n_{\text {middle }}$ tertile 267, 33\%). To determine the effect that the abstracted dimension (i.e. 'food purchasing behaviour') had on morphological variables, the tertiles were stratified by both sex and 10-year age groups (20-29 years, 30-39 years, 40-49 years, 50-59 years, 60 years or older). Two analyses were conducted to evaluate the use of MCA-determined tertiles in assessing obesity. First, ANOVA was used to

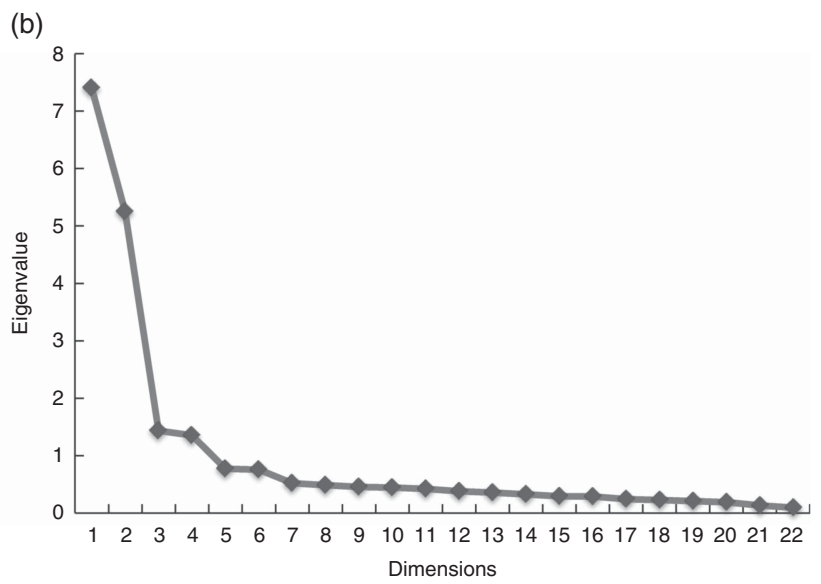

Fig. 1 Scree plots of eigenvalues for (a) dimensions established by the first multiple correspondence analysis (MCA) with seventythree variables and (b) dimensions established by the second MCA with twenty-two variables, identified from an extensive behavioural survey administered to Vanuatu adults ( $n$ 810) aged 20-85 years. A dimension's eigenvalue is a measure of the amount of variance for which it accounts 
Correlations $\left(\eta^{2}\right)$ with Dimensions 1 and 2

Variable

1. Do you buy oil in a store? $\mathrm{Y} / \mathrm{N}$

2. Do you buy salt in a store? $\mathrm{Y} / \mathrm{N}$

3. Do you buy sugar in a store? $\mathrm{Y} / \mathrm{N}$

4. Do you buy rice in a store? $\mathrm{Y} / \mathrm{N}$

5. Do you buy tinned fish/meat in a store? $\mathrm{Y} / \mathrm{N}$

6. Do you buy noodles in a store? $\mathrm{Y} / \mathrm{N}$

7. Do you buy peanut butter in a store? $\mathrm{Y} / \mathrm{N}$

8. Do you buy gato (doughnuts) in a store? $\mathrm{Y} / \mathrm{N}$

9. Do you buy fresh meat in a store? $\mathrm{Y} / \mathrm{N}$

10. Do you buy butter in a store? $\mathrm{Y} / \mathrm{N}$

11. Do you buy food in a store? $\mathrm{Y} / \mathrm{N}$

12. Do you buy biscuits in a store? $\mathrm{Y} / \mathrm{N}$

13. Do you own a computer/laptop? $Y / N$

14. Do you use the Internet at home? $Y / N$

15. Do you use the Internet to find information? $\mathrm{Y} / \mathrm{N}$

16. Do you use the Internet to listen to music? Y/N

17. Do you use the Internet to watch videos? $Y / N$

18. Do you use the Internet to chat and messaging? $Y / N$

19. Do you use the Internet to read news? Y/N

20. Do you use the Internet to check email? Y/N

21. Do you use the Internet to find new friends? $\mathrm{Y} / \mathrm{N}$

22. Do you use the Internet at school? Y/N

Dimension

$\begin{array}{cc}1 & 2 \\ 0.668 & 0.083\end{array}$

$0.668 \quad 0.083$

$\begin{array}{ll}0.652 & 0.116 \\ 0.647 & 0.109\end{array}$

$\begin{array}{ll}0.647 & 0.109 \\ 0.605 & 0.109\end{array}$

$\begin{array}{ll}0.599 & 0.102\end{array}$

$0.502 \quad 0.064$

$0.499 \quad 0.037$

$\begin{array}{ll}0.460 & 0.042\end{array}$

$0.459 \quad 0.019$

$0.417 \quad 0.022$

$\begin{array}{ll}0.415 & 0.038\end{array}$

$0.377 \quad 0.064$

$0.146 \quad 0.415$

$0.143 \quad 0.573$

$\begin{array}{ll}0.134 & 0.539\end{array}$

$\begin{array}{ll}0.134 & 0.539 \\ 0.123 & 0.569\end{array}$

$0.123 \quad 0.569$

$0.112 \quad 0.549$

$\begin{array}{ll}0.109 & 0.357 \\ 0.101 & 0.470\end{array}$

$0.097 \quad 0.316$

$0.072 \quad 0.283$

$0.065 \quad 0.376$

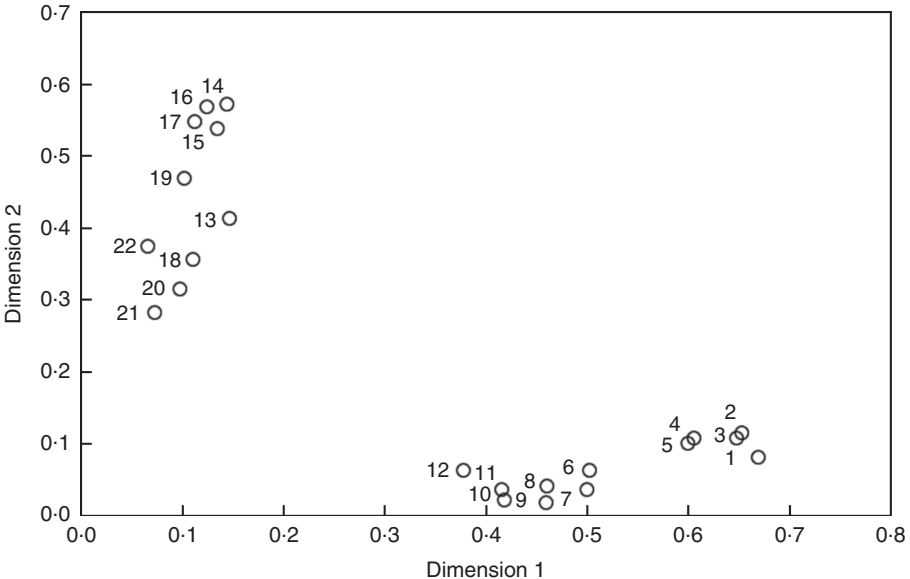

Fig. 2 The $\eta^{2}$ values related to two dimensions determined by the second multiple correspondence analysis for twenty-two variables, identified from an extensive behavioural survey administered to Vanuatu adults ( $n$ 810) aged 20-85 years. Dimension 1 accounts for $33.6 \%$ of the variance in this analysis, while Dimension 2 accounts for $22.8 \%$

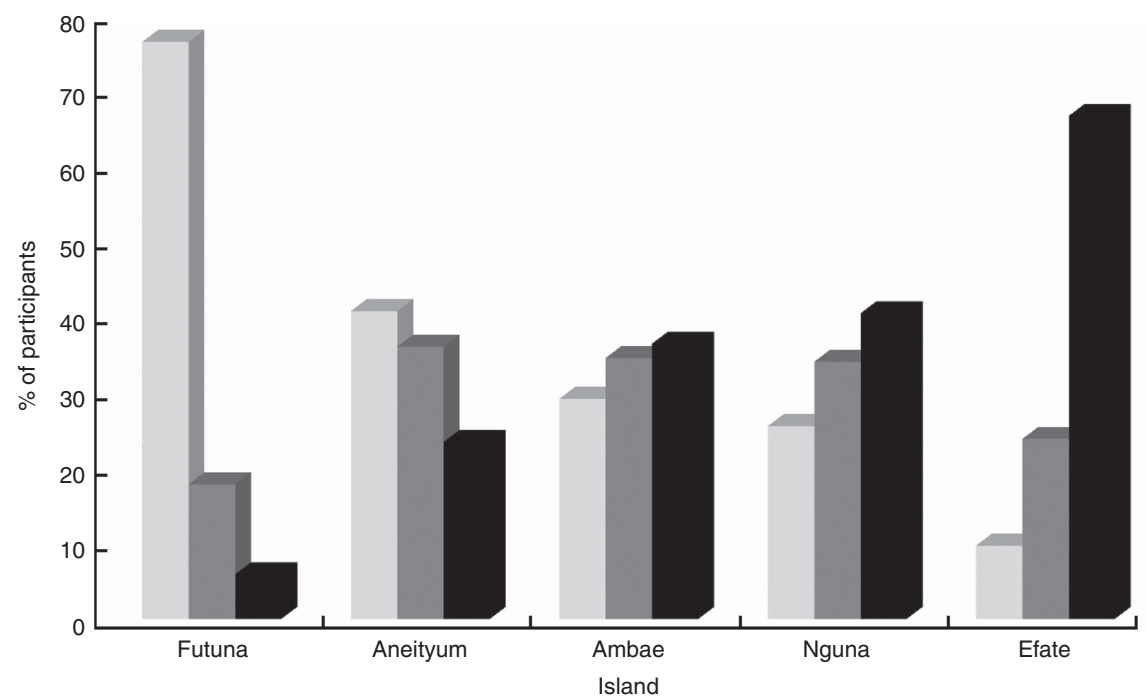

Fig. 3 Distribution of food purchasing tertiles ( $\square$, lower tertile; $\square$, middle tertile; $\square$, upper tertile) by island (males and females combined) among Vanuatu adults $(n 810)$ aged $20-85$ years. For differences in distribution of men and women in the three tertiles among the six study islands, $x^{2}=198.369(P<0.001)$

determine if morphological indicators of overweight/ obesity differed significantly between tertiles and/or age groups for each sex. Statistical significance was determined at $P<0.05$ or greater. Second, OR and their $95 \% \mathrm{CI}$ were computed to evaluate whether individuals in the upper tertile identified by MCA were more likely to exhibit obesity than those in the lower two tertiles. For this purpose, individuals were classified as obese if their subscapular skinfold, triceps skinfold or BMI measurements fell above the 75th percentile, using US National Health Statistics standards for adults ${ }^{(33)}$; or if their \%BF fell above the obesity level predicted by equations based on $\mathrm{BMI}^{(34)}$. Since obesity classification was based on age- and sexspecific parameters (i.e. whether an individual fell above the 75 th percentile for the US National Health Statistics standards for their age and sex group), there was no need to adjust for age and sex differences; and all individuals in the sample were combined for this analysis. All statistical analyses were performed using the statistical software package IBM SPSS Statistics for Windows version 21.0.

\section{Results}

Figure 3 shows the distribution of tertiles by island (males and females combined). The overwhelming majority of individuals on Futuna $(76.3 \%)$ fell into the lower tertile (i.e. were least likely to purchase food items at a store), while on Efate the overwhelming majority of individuals (66.5\%) fell into the upper tertile (i.e. were most likely to 
purchase food items at a store). On other islands, there was a more uniform distribution of individuals in the three tertiles. As is obvious from Fig. 3, the difference between observed and expected values for the distribution of tertiles among the islands is highly significant $\left(\chi^{2}=198.369, \mathrm{df}=8, P<0 \cdot 001\right)$. Over all ages, the proportion of males in the upper tertile was greater than the proportion of females, while the proportion of females in the lower and middle tertiles was greater than the proportion of males $\left(\chi^{2}\right.$ over all ages $\left.=16 \cdot 778, P<0 \cdot 001\right)$; see the online supplementary material, Supplemental Fig. 1. Differences in proportions of males and females in the three tertiles were statistically significant in the age categories 20-29 years $\left(\chi^{2}=10 \cdot 581, P=0.005\right)$ and 30-39 years $\left(\chi^{2}=7 \cdot 047, P=0.030\right)$; but not in the three older age categories.

Tables 1 and 2 show means and SD of three general measures of adiposity (weight, BMI, \%BF) for men and women, arranged by 10-year age groups. Over all ages, weight, BMI and \%BF were significantly greater among both men and women who were most likely to purchase food items at a store compared with those who purchased few or no food items at stores. In each case, post hoc analysis indicated that the differences between the tertile that was most likely to purchase food at a store and the tertile that was least likely to purchase food at a store were highly significant. Nevertheless, the pattern of tertile differences in $\mathrm{BMI}$ and \%BF was not uniform in all age groups. Among men and women in their 20s and 30s, food purchasing behaviour was not associated with major differences in BMI or \%BF. Among women, the most significant differences between tertiles occurred among those in the age category 40-49 years. Differences between tertiles diminished among older women, particularly those aged 60 years or older. Among men, the greatest differences between tertiles occurred among those in the oldest age group.

This pattern is dramatically illustrated when skinfolds are compared between tertiles. Tables 3 and 4 show marginal means across the age groups for triceps skinfolds, subscapular skinfolds and suprailliac skinfolds for men and women, adjusted for differences in height and weight (both significant covariates of skinfolds). Among men, mean adjusted triceps skinfolds did not differ significantly between food purchasing tertiles; but differences in measures of trunk fat (adjusted subscapular and suprailliac skinfolds) were highly significant. Both measures increased much more substantially with age among men who were most likely to purchase food at stores compared with men in the middle and lower tertiles. Among women, adjusted mean triceps skinfolds differed significantly among the tertiles, but differences among tertiles did not vary significantly with age. Thus, women in the upper tertile have higher triceps skinfolds than women in the other two tertiles, regardless of age. On the other hand, there were no significant differences in either subscapular or suprailliac skinfolds associated with food purchasing behaviour. However, this was mostly due to the similarity in adjusted skinfold measures, regardless of food purchasing behaviour, among women aged 60 years or older. Among women under the age of 60 years, adjusted subscapular and suprailliac skinfolds were significantly greater among those who were most likely to buy food at stores.

Table 1 Means and SD of weight and body fat measures among Vanuatu men aged 20-85 years by food purchasing tertile and age group

\begin{tabular}{|c|c|c|c|c|c|c|c|}
\hline \multirow[b]{2}{*}{ Food purchasing tertile/Age group } & \multirow[b]{2}{*}{$n$} & \multicolumn{2}{|c|}{ Weight (kg) } & \multicolumn{2}{|c|}{ BMI $\left(\mathrm{kg} / \mathrm{m}^{2}\right)$} & \multicolumn{2}{|c|}{$\%$ Body fat } \\
\hline & & Mean & SD & Mean & SD & Mean & SD \\
\hline \multicolumn{8}{|l|}{ High } \\
\hline $20-29$ years & 41 & $65 \cdot 8$ & $7 \cdot 4$ & $23 \cdot 0$ & $2 \cdot 0$ & $15 \cdot 4$ & 3.5 \\
\hline $30-39$ years & 41 & $76 \cdot 3$ & $14 \cdot 2$ & $25 \cdot 9$ & $4 \cdot 1$ & $20 \cdot 8$ & 6.9 \\
\hline 40-49 years & 37 & $78 \cdot 7$ & $17 \cdot 1$ & $27 \cdot 3$ & $5 \cdot 3$ & $23 \cdot 2$ & 8.8 \\
\hline $50-59$ years & 29 & $74 \cdot 1$ & 13.5 & 26.5 & 4.3 & $22 \cdot 8$ & $7 \cdot 6$ \\
\hline 60 years or older & 14 & 74.9 & $11 \cdot 3$ & $26 \cdot 9$ & 3.5 & $23 \cdot 6$ & $7 \cdot 0$ \\
\hline \multicolumn{8}{|l|}{ Medium } \\
\hline 20-29 years & 21 & $65 \cdot 8$ & $5 \cdot 3$ & $23 \cdot 2$ & $2 \cdot 0$ & $15 \cdot 5$ & 3.7 \\
\hline $30-39$ years & 26 & $70 \cdot 8$ & $12 \cdot 7$ & $24 \cdot 6$ & $3 \cdot 1$ & $17 \cdot 8$ & $5 \cdot 2$ \\
\hline 40-49 years & 31 & $75 \cdot 6$ & $12 \cdot 4$ & $26 \cdot 3$ & 3.8 & 21.4 & 6.9 \\
\hline $50-59$ years & 15 & $68 \cdot 0$ & 10.5 & $25 \cdot 1$ & 3.8 & $19 \cdot 9$ & $6 \cdot 2$ \\
\hline 60 years or older & 18 & 67.4 & $9 \cdot 8$ & $25 \cdot 9$ & 3.0 & $21 \cdot 0$ & $6 \cdot 0$ \\
\hline \multicolumn{8}{|l|}{ Low } \\
\hline $20-29$ years & 32 & $66 \cdot 0$ & $8 \cdot 3$ & 23.4 & $2 \cdot 6$ & $15 \cdot 6$ & 4.5 \\
\hline $30-39$ years & 25 & $70 \cdot 3$ & $11 \cdot 0$ & $24 \cdot 6$ & $2 \cdot 9$ & $17 \cdot 9$ & 4.9 \\
\hline $40-49$ years & 23 & $72 \cdot 8$ & 7.8 & 25.4 & $3 \cdot 1$ & $20 \cdot 0$ & $5 \cdot 3$ \\
\hline $50-59$ years & 19 & 73.0 & $12 \cdot 7$ & $25 \cdot 9$ & $3 \cdot 3$ & $19 \cdot 9$ & $6 \cdot 1$ \\
\hline 60 years or older & 26 & 64.0 & 11.5 & $24 \cdot 4$ & 3.9 & $17 \cdot 7$ & $6 \cdot 2$ \\
\hline$F$ (food purchasing tertile) & & \multicolumn{2}{|c|}{$6 \cdot 640^{\star \star \star}$} & \multicolumn{2}{|c|}{$3.989^{\star}$} & \multicolumn{2}{|c|}{$7 \cdot 755^{\star \star \star}$} \\
\hline$F$ (age group) & & \multicolumn{2}{|c|}{$8 \cdot 505^{\star \star \star}$} & \multicolumn{2}{|c|}{$9 \cdot 957^{\star \star *}$} & \multicolumn{2}{|c|}{$13.031^{\star * *}$} \\
\hline
\end{tabular}

${ }^{\star} P<0.05,{ }^{* \star *} P<0.001$. 
Table 2 Means and SD of weight and body fat measures among Vanuatu women aged 20-85 years by food purchasing tertile and age group

\begin{tabular}{|c|c|c|c|c|c|c|c|}
\hline \multirow[b]{2}{*}{ Food purchasing tertile/Age group } & \multirow[b]{2}{*}{$n$} & \multicolumn{2}{|c|}{ Weight (kg) } & \multicolumn{2}{|c|}{ BMI $\left(\mathrm{kg} / \mathrm{m}^{2}\right)$} & \multicolumn{2}{|c|}{$\%$ Body fat } \\
\hline & & Mean & SD & Mean & SD & Mean & SD \\
\hline \multicolumn{8}{|l|}{ High } \\
\hline 20-29 years & 28 & $60 \cdot 8$ & $14 \cdot 3$ & $24 \cdot 3$ & $5 \cdot 2$ & $29 \cdot 3$ & $9 \cdot 6$ \\
\hline 30-39 years & 29 & $62 \cdot 0$ & $12 \cdot 7$ & $25 \cdot 6$ & $5 \cdot 6$ & $30 \cdot 2$ & $9 \cdot 1$ \\
\hline 40-49 years & 25 & $77 \cdot 8$ & 18.5 & $30 \cdot 6$ & $6 \cdot 7$ & $36 \cdot 8$ & $9 \cdot 3$ \\
\hline $50-59$ years & 20 & $69 \cdot 7$ & 13.7 & $28 \cdot 2$ & $5 \cdot 4$ & 34.6 & $6 \cdot 8$ \\
\hline 60 years or older & 12 & 68.3 & $16 \cdot 3$ & 28.4 & $5 \cdot 6$ & $32 \cdot 8$ & $8 \cdot 3$ \\
\hline \multicolumn{8}{|l|}{ Medium } \\
\hline 20-29 years & 44 & 57.5 & $8 \cdot 6$ & $22 \cdot 9$ & 3.0 & $26 \cdot 6$ & $6 \cdot 1$ \\
\hline 30-39 years & 45 & $65 \cdot 0$ & $15 \cdot 2$ & $25 \cdot 8$ & $5 \cdot 7$ & $30 \cdot 8$ & $9 \cdot 7$ \\
\hline 40-49 years & 29 & $66 \cdot 7$ & $12 \cdot 3$ & $26 \cdot 5$ & 4.7 & $31 \cdot 7$ & $7 \cdot 7$ \\
\hline $50-59$ years & 20 & $66 \cdot 4$ & $22 \cdot 8$ & $27 \cdot 9$ & $9 \cdot 2$ & 30.5 & 11.5 \\
\hline 60 years or older & 18 & 64.5 & 13.4 & $27 \cdot 1$ & $6 \cdot 0$ & $31 \cdot 2$ & $8 \cdot 3$ \\
\hline \multicolumn{8}{|l|}{ Low } \\
\hline 20-29 years & 46 & $60 \cdot 8$ & $10 \cdot 0$ & $24 \cdot 7$ & $3 \cdot 6$ & $29 \cdot 3$ & $6 \cdot 1$ \\
\hline 30-39 years & 32 & $61 \cdot 1$ & $12 \cdot 5$ & $24 \cdot 3$ & 4.5 & $28 \cdot 3$ & 8.2 \\
\hline 40-49 years & 23 & $60 \cdot 3$ & $9 \cdot 3$ & $24 \cdot 2$ & 3.6 & $27 \cdot 6$ & $8 \cdot 1$ \\
\hline $50-59$ years & 27 & 63.5 & 9.9 & $27 \cdot 1$ & 4.5 & 31.0 & $7 \cdot 4$ \\
\hline 60 years or older & 14 & $56 \cdot 6$ & $10 \cdot 7$ & $24 \cdot 3$ & $4 \cdot 1$ & $26 \cdot 9$ & 9.4 \\
\hline$F$ (food purchasing tertile) & & \multicolumn{2}{|c|}{$8 \cdot 265^{\star \star \star}$} & \multicolumn{2}{|c|}{$6 \cdot 651^{\star \star}$} & \multicolumn{2}{|c|}{$6.902^{\star \star \star}$} \\
\hline$F$ (age group) & & \multicolumn{2}{|c|}{$5 \cdot 624^{\star \star}$} & \multicolumn{2}{|c|}{$7 \cdot 565^{\star \star \star}$} & \multicolumn{2}{|c|}{$3 \cdot 184^{\star}$} \\
\hline
\end{tabular}

${ }^{\star} P<0.05,{ }^{* *} P<0.01,{ }^{* \star *} P<0.001$.

Table 3 Means and SE of skinfold measurements, adjusted for differences in height and weight, among Vanuatu men aged 20-85 years by food purchasing tertile and age group

\begin{tabular}{|c|c|c|c|c|c|c|c|}
\hline \multirow[b]{2}{*}{ Food purchasing tertile/Age group } & \multirow[b]{2}{*}{$n$} & \multicolumn{2}{|c|}{ Triceps skinfold (mm) } & \multicolumn{2}{|c|}{ Subscapular skinfold (mm) } & \multicolumn{2}{|c|}{ Suprailliac skinfold (mm) } \\
\hline & & Mean & SE & Mean & SE & Mean & SE \\
\hline \multicolumn{8}{|l|}{ High } \\
\hline 20-29 years & 28 & 9.8 & 0.7 & $17 \cdot 3$ & 0.9 & $15 \cdot 6$ & $1 \cdot 2$ \\
\hline $30-39$ years & 29 & 11.6 & 0.7 & $19 \cdot 1$ & 0.9 & $19 \cdot 8$ & 1.2 \\
\hline $40-49$ years & 25 & $10 \cdot 3$ & 0.7 & $21 \cdot 0$ & 1.0 & 22.5 & 1.3 \\
\hline $50-59$ years & 20 & $11 \cdot 3$ & 0.8 & $22 \cdot 2$ & 1.1 & $22 \cdot 7$ & 1.4 \\
\hline 60 years or older & 12 & $15 \cdot 1$ & 1.2 & 23.5 & 1.5 & $26 \cdot 6$ & $2 \cdot 0$ \\
\hline \multicolumn{8}{|l|}{ Medium } \\
\hline $20-29$ years & 44 & 9.6 & 1.0 & $15 \cdot 6$ & 1.3 & 13.0 & 1.7 \\
\hline $30-39$ years & 45 & 10.5 & 0.9 & $16 \cdot 6$ & 1.1 & $18 \cdot 0$ & 1.5 \\
\hline 40-49 years & 29 & $10 \cdot 9$ & 0.8 & $17 \cdot 7$ & 1.0 & $17 \cdot 7$ & 1.4 \\
\hline $50-59$ years & 20 & 13.6 & 1.2 & $20 \cdot 9$ & 1.5 & $18 \cdot 6$ & $2 \cdot 0$ \\
\hline 60 years or older & 18 & $10 \cdot 4$ & 1.1 & 19.9 & 1.4 & $19 \cdot 1$ & 1.8 \\
\hline \multicolumn{8}{|l|}{ Low } \\
\hline 20-29 years & 46 & $10 \cdot 3$ & 0.8 & $15 \cdot 8$ & 1.0 & $13 \cdot 3$ & 1.4 \\
\hline $30-39$ years & 32 & $10 \cdot 5$ & 0.9 & $16 \cdot 7$ & $1 \cdot 2$ & $18 \cdot 3$ & 1.5 \\
\hline $40-49$ years & 23 & 11.6 & 0.9 & $16 \cdot 2$ & 1.2 & 14.0 & 1.6 \\
\hline $50-59$ years & 27 & 11.7 & $1 \cdot 1$ & $18 \cdot 4$ & 1.4 & $17 \cdot 9$ & 1.8 \\
\hline 60 years or older & 14 & $10 \cdot 7$ & 0.9 & $18 \cdot 2$ & 1.2 & 18.0 & 1.5 \\
\hline$F$ (food purchasing tertile) & & \multirow{2}{*}{\multicolumn{2}{|c|}{$\begin{array}{l}0.822 \\
2.777^{*}\end{array}$}} & \multirow{2}{*}{\multicolumn{2}{|c|}{$\begin{array}{r}12 \cdot 428^{\star \star \star} \\
6 \cdot 450^{\star \star \star}\end{array}$}} & \multirow{2}{*}{\multicolumn{2}{|c|}{$\begin{array}{r}16 \cdot 096^{\star \star \star} \\
8.535^{\star \star \star}\end{array}$}} \\
\hline$F$ (age group) & & & & & & & \\
\hline
\end{tabular}

${ }^{\star} P<0.05,{ }^{* \star \star} P<0.001$

The OR presented in Table 5 show that, for each measure of body fat, but particularly for skinfolds and BMI, Vanuatu individuals in the highest food purchasing tertile were significantly more likely to be above the 75 th percentile compared with individuals in the lower two tertiles. Thus, the identification of these tertiles by MCA is useful for identifying individuals who are likely to be obese.

\section{Discussion}

In the present study, MCA was used to explore the responses to a set of questions designed to assess modernization status. The goal was to identify behavioural characteristics associated with modernization that were relevant to variation in overweight/obesity among 
Table 4 Means and SE of skinfold measurements, adjusted for differences in height and weight, among Vanuatu women aged 20-85 years by food purchasing tertile and age group

\begin{tabular}{|c|c|c|c|c|c|c|c|}
\hline \multirow[b]{2}{*}{ Food purchasing tertile/Age group } & \multirow[b]{2}{*}{$n$} & \multicolumn{2}{|c|}{ Triceps skinfold (mm) } & \multicolumn{2}{|c|}{ Subscapular skinfold (mm) } & \multicolumn{2}{|c|}{ Suprailliac skinfold (mm) } \\
\hline & & Mean & SE & Mean & SE & Mean & SE \\
\hline \multicolumn{8}{|l|}{ High } \\
\hline 20-29 years & 28 & $19 \cdot 8$ & 1.0 & 23.7 & $1 \cdot 1$ & $26 \cdot 4$ & 1.5 \\
\hline $30-39$ years & 29 & $22 \cdot 1$ & 1.0 & 24.7 & $1 \cdot 1$ & 24.7 & 1.5 \\
\hline $40-49$ years & 25 & $21 \cdot 2$ & $1 \cdot 1$ & $26 \cdot 2$ & 1.2 & 25.5 & 1.6 \\
\hline 50-59 years & 20 & $22 \cdot 8$ & $1 \cdot 2$ & 24.5 & 1.4 & $29 \cdot 7$ & $1 \cdot 8$ \\
\hline 60 years or older & 12 & $20 \cdot 7$ & 1.5 & $20 \cdot 7$ & 1.7 & $25 \cdot 5$ & $2 \cdot 3$ \\
\hline \multicolumn{8}{|l|}{ Medium } \\
\hline $20-29$ years & 44 & $19 \cdot 9$ & 0.8 & $20 \cdot 2$ & 0.9 & $23 \cdot 3$ & 1.2 \\
\hline $30-39$ years & 45 & $20 \cdot 8$ & 0.8 & 23.4 & 0.9 & $25 \cdot 9$ & 1.2 \\
\hline 40-49 years & 29 & $20 \cdot 7$ & 1.0 & 24.5 & $1 \cdot 1$ & $25 \cdot 3$ & $1 . \overline{5}$ \\
\hline $50-59$ years & 20 & $21 \cdot 7$ & 1.2 & $22 \cdot 8$ & 1.4 & $25 \cdot 1$ & 1.8 \\
\hline 60 years or older & 18 & $16 \cdot 9$ & 1.3 & $21 \cdot 7$ & 1.4 & $25 \cdot 7$ & 1.9 \\
\hline \multicolumn{8}{|l|}{ Low } \\
\hline $20-29$ years & 46 & 19.5 & 0.8 & $21 \cdot 2$ & 0.9 & 23.5 & 1.2 \\
\hline $30-39$ years & 32 & 19.5 & 0.9 & 22.5 & 1.0 & $22 \cdot 8$ & 1.4 \\
\hline $40-49$ years & 23 & $18 \cdot 9$ & $1 \cdot 1$ & 23.3 & 1.2 & 23.2 & 1.6 \\
\hline $50-59$ years & 27 & $18 \cdot 9$ & 1.0 & 21.3 & 1.2 & $24 \cdot 1$ & 1.6 \\
\hline 60 years or older & 14 & $18 \cdot 3$ & 1.4 & 23.0 & 1.6 & $25 \cdot 9$ & $2 \cdot 1$ \\
\hline$F$ (food purchasing tertile) & & \multicolumn{2}{|c|}{$5 \cdot 271^{\star \star}$} & \multicolumn{2}{|c|}{2.675} & \multicolumn{2}{|c|}{2.573} \\
\hline$F$ (age group) & & \multicolumn{2}{|c|}{1.182} & \multicolumn{2}{|c|}{$3 \cdot 388^{\star *}$} & \multicolumn{2}{|c|}{0.716} \\
\hline
\end{tabular}

${ }^{* *} P<0.01$.

\begin{tabular}{|c|c|c|c|}
\hline Measure of obesity & OR & $95 \% \mathrm{Cl}$ & Significance (two-sided) \\
\hline Subscapular skinfold & & & \\
\hline $\begin{array}{l}\geq 75 \text { th percentile } \\
\text { Triceps skinfold }\end{array}$ & 2.028 & $1.480,2.781$ & $<0.001$ \\
\hline$\underset{\mathrm{BMI}}{\geq 75 \text { th percentile }}$ & $1 \cdot 817$ & $1 \cdot 208,2 \cdot 773$ & 0.004 \\
\hline $\begin{array}{l}\geq 75 \text { th percentile } \\
\% \text { Body fat }\end{array}$ & $2 \cdot 020$ & $1 \cdot 218,3.351$ & 0.006 \\
\hline Associated with $\mathrm{BMI} \geq 30.0 \mathrm{~kg} / \mathrm{m}^{2}$ & $1 \cdot 708$ & $1.124,2.595$ & 0.012 \\
\hline
\end{tabular}

Vanuatu adults, based on the responses to seventy-three yes/no questions. In this case, the most significant 'explanatory' vector identified by MCA (i.e. the vector associated with the greatest proportion of variance defined by the survey questions) included individual responses to twelve questions related to buying food at a store. The position of each individual on the numerical scale derived from this vector ranged from those who answered 'yes' to all twelve questions to those who answered 'no' to all twelve questions. Based on their position on this scale, individuals were divided into three tertiles that differed in food purchasing behaviour. Subsequent analysis indicated that individuals in the tertile most likely to purchase food at stores were significantly heavier and fatter than individuals in the tertile least likely to purchase food at stores. Furthermore, the odds of exhibiting BMI and skinfold values above the 75th US percentile were doubled among individuals in the highest tertile relative to those in other two tertiles. Thus, as an exploratory quantitative method,
MCA has the ability to position individuals on an axis that is related to 'modernization' status and correlate that position with individual differences in morphology and health.

It is noteworthy that modernization status in Vanuatu is most strongly reflected in food purchasing behaviour, rather than in any of the other activities included in the seventy-three questions analysed by MCA. This may be related to the inclusion of questions concerning what cash was spent on (e.g. travel, recreational activities, ownership of appliances, ownership and use of electronic devices, etc., in addition to food purchases at stores), rather than how much cash was earned. Consequently, the modernization status elicited by our survey is likely associated with differences in the use of cash. Since individual variation in food purchases is greater than variation in the other uses of cash, it was identified as the most significant explanatory component in our modernization profile. 
That being said, it is not surprising that food purchases at stores are associated with the risk of obesity and overweight. The food purchases covered by questions in the survey consisted of high-energy dietary supplements (sugar, oil), energy-dense processed foods (peanut butter, tinned meat, tinned fish) and refined grains (rice, noodles). The apparently greater access to these types of foods among individuals in the upper tertile in the present study corresponds to a worldwide shift in the food system caused by economic growth and fuelled by international organizations that promote trade between developed and developing nations ${ }^{(35)}$. In the Pacific, trade agreements and international investments have promoted an increasing availability of energy-dense, nutritionally poor processed foods ${ }^{(36-39)}$. Vanuatu is a signatory of the Pacific Island Countries Trade Agreement, which requires member countries to reduce tariffs and other barriers to imports ${ }^{(37)}$, and the World Trade Organization, which similarly limits trade barriers ${ }^{(36)}$. Thus, it is not surprising that imported foods more than tripled between 2005 and $2015^{(40)}$ - although perhaps to a somewhat lesser extent than in other Pacific Island nations ${ }^{(37)}$. However, food imports into Vanuatu are currently growing at a faster pace than the world average ${ }^{(41)}$.

Pacific Island studies have generally shown that increases in overweight/obesity are linked to economic development ${ }^{(3,6,7,14,16,42)}$, economic status ${ }^{(5,15)}$ and/or sociocultural factors ${ }^{(10,11)}$. However, the relatively few studies that have assessed the effect of specific dietary components on overweight/obesity in Pacific Island countries do not consistently demonstrate an association between Westernized diets and obesity. An initial study in Samoa ${ }^{(42)}$ and a study in Vanuatu ${ }^{(14)}$ indicate that even modest incorporation of Western foods in the diet can contribute to increased risk of obesity. On the other hand, a more recent study in Samoa ${ }^{(16)}$ has reported that a diet that contains a mix of modern and traditional dietary elements is associated with a greater risk of obesity than diets that are totally modern. Among migrants from Bougainville to Port Moresby, there was no association between obesity and the inclusion of Western food items in the diet; but variation in measures of obesity were significantly associated with differences in activity ${ }^{(43)}$.

These studies indicate that the relationship between economic development, dietary choice and obesity in the developing nations of the South Pacific may be complicated by a number of confounding factors. Among them is the uneven penetration of the cash economy ${ }^{(37)}$. In Vanuatu, large-scale enterprises involved in the distribution of imported foods (supermarkets, cafés/bars, fast-food restaurants and convenience stores) exist only on Efate. However, even on Efate, many people live in rural settlements without consistent access to mass distribution outlets. On less developed Vanuatu islands, biscuits, chips, candy, gato, tinned meat or tinned fish, and sodas or sometimes beer are available in small stores, run (often on an irregular basis) by local entrepreneurs. Thus, the non-uniform availability of food to purchase likely contributes to differences in the prevalence of obesity between and within islands ${ }^{(13,14)}$.

Nevertheless, it is important to note that, despite island differences in food distribution infrastructure, our MCA links the purchase of processed foods at stores to overweight/obesity. This finding is potentially significant in evaluating various initiatives undertaken to combat obesity in Vanuatu. At present, national government strategies seem to focus on regulating the energy-dense content of dietary staples, on taxing soft drinks, on mandating nutrition content labelling and on a variety of public education programmes ${ }^{(38,44)}$. There also have been initiatives at the local level, aimed at banning all junk foods ${ }^{(45)}$. Our results indicate that the most effective approaches in the short term are likely those that affect availability of junk foods at local stores and shops. In this regard, labelling or taxation strategies are less likely to have an impact as long as junk foods are available. On the other hand, government proposals to elevate awareness of good dietary practices by initiating nutrition education programmes may have greater success over the long run.

In addition to island differences in mercantile infrastructure, there appear to exist age and gender differences in the way people use cash to purchase food items in stores on all islands. Our study indicates that buying food at stores is less likely to be associated with obesity among younger individuals than among older individuals. Among younger $\mathrm{Ni}$ Vanuatu, an active lifestyle regardless of differences in the degree to which individuals purchase store-bought foods may limit the effect of dietary differences, similar to the pattern noted among young migrants to Port Moresby ${ }^{(43)}$. The increased risk of overweight/obesity among older adults who purchase food at stores suggests that attitudes about food purchases (possibly including cultural factors influencing the type of dietary items on which cash is spent ${ }^{(12)}$ ) may differ according to age and also contribute to the risk of overweight/obesity. Gender appears to introduce a further complication. Among women, tertile differences in measures of body fat - particularly adjusted skinfold measurements - peak in the 40-49 years age group, and then diminish with age. This could be related to a more uniform activity pattern among older women regardless of the extent to which they purchase food at stores. Among similarly modernizing groups in the Solomon Islands, older women purchased food at stores for others in their families while still maintaining strenuous traditional work patterns ${ }^{(46)}$. Among older men in Vanuatu, on the other hand, tertile differences in measures of body fat increase with age. Perhaps, like older men in the Solomon Islands ${ }^{(46)}$, exercise capacity was inversely proportional to modernization status - thereby causing differences in obesity risk to be associated with both increased purchases of food in stores and reduced activity.

An important limitation of the present study is that the questions included in the survey do not indicate how 
much food is purchased at stores, or how frequently it is purchased and consumed. Since both eating pattern and the frequency of processed food purchases may vary by age and sex groups, there is a clear need for follow-up research to clarify dietary consumption patterns. While we did not directly measure activity pattern, several questions about activity types were included in the survey. Differences in the responses to these questions did not map to tertiles associated with food purchasing behaviour and were not identified by MCA as contributing significantly to overall behavioural variability. An additional limitation is the cross-sectional nature of the survey. The age differences in the pattern of food purchases and overweight/ obesity suggest a cohort effect. However, while average weight and measures of adiposity among individuals who are most likely to purchase food at stores increase with age, and therefore might be connected with the long-term consumption of imported foods, this cannot be confirmed without further longitudinal studies.

Our estimates of population sizes, while admittedly crude, indicate that we obtained samples that accurately reflected the age and sex structure of the larger of populations on three islands and the local region on a fourth. Our sampling strategy on the fifth island, Efate, was admittedly not large enough to represent the island with the greatest engagement in the cash economy. Thus, our results are probably best applied to the rural Vanuatu population - $75 \%$ of the total ${ }^{(27)}$. Nevertheless, our sampling strategy, aimed at including as many individuals from the target populations as possible, is probably the best method of obtaining representative samples in areas of the world where local demographic data are not easily obtainable.

Even though the survey upon which the analysis was based was not designed with the expectation that MCA would be performed, MCA proved to be effective in identifying behaviours linked to modernization that were significantly related to obesity risk. This suggests that MCA could be applied to existing Vanuatu databases such as those generated by the Vanuatu National Statistics Office. These data include nationwide responses to questionnaires about a variety of individual behavioural characteristics, broken down by region, sex and age ${ }^{(29,47)}$, and cover Efate and other urban areas, as well more remote locations. MCA of these data could potentially identify aggregate behaviours that might be related to obesity in different segments of the population and could be used to design specifically targeted initiatives to improve nutrition and reduce obesity.

\section{Conclusion}

The present study indicates that MCA represents an important addition to the list of techniques that can be used to identify behaviours that are associated with the impact of modernization on overweight/obesity. Using categorical variables, MCA is able to identify individuallevel patterns of behavioural variability within and among diverse social and geographic groups. Out of seventy-three questions concerning a wide range of behaviours associated with modernization, MCA identified twelve questions related to food purchases at stores that explained the greatest individual variance. The simple 'yes/no' responses to these twelve questions about food purchasing were sufficiently robust to identify individuals at risk for overweight/obesity regardless of island of residence, rural or urban location, or the presence/absence of major food distribution infrastructure. Therefore, MCA may be particularly useful in identifying categorical variables associated with obesity risk in research among modernizing populations when the conditions of study groups are either unknown or potentially diverse, or when funds, manpower and/or time are limited.

\section{Acknowledgements}

Acknowledgements: The authors wish to thank all the participants. Financial support: This work was supported by the Wenner-Gren Foundation for Anthropological Research (R.M.G., grant number 8301), by a Grant-in-Aid and a Summer Research Fellowship from Temple University (C.A.W.), and by the Harpur College Grants in Support of Research, Scholarship and Creative Work at Binghamton University (J.K.L.). The Wenner-Gren Foundation, Binghamton University and Temple University had no role in the design, analysis or writing of this article. Conflict of interest: None. Authorship: R.M.G. and J.K.L. designed the research; C.A.W., K.M.O., K.N.D., C.S., A.P., H.S., G.L., C.W.C., A.K., J.K.L. and R.M.G. conducted the research and collected the data; A.V.H. and C.A.W. analysed the data and performed statistical analyses; C.A.W. and A.V.H. wrote paper; C.A.W. and A.V.H. had primary responsibility for final content; all authors edited and reviewed and approved the final manuscript. Ethics of buman subject participation: This study was conducted according to the guidelines laid down in the Declaration of Helsinki and all procedures involving human subjects were approved by the Ethics Committee of Temple University (Temple University Institutional Review Board, protocol number 13827) and by the Ethics Committee of Binghamton University (Binghamton University Institutional Review Board, protocol number 1578-10) before the commencement of the study. Permission to conduct research in-country was granted by the Vanuatu Ministry of Health. In each village where the study took place, the research protocol and aims were explained to local chiefs and, at a general meeting, to the community at large. Informed consent was verbally obtained from each adult 
participant and witnessed by a member of the research team, following a protocol approved by the Institutional Review Boards of Binghamton University and Temple University, and by the Vanuatu Ministry of Health.

\section{Supplementary material}

To view supplementary material for this article, please visit https://doi.org/10.1017/S1368980019000302

\section{References}

1. Baker PT, Hanna JM \& Baker TS (editors) (1986) The Changing Samoans: Behavior and Health in Transition. New York: Oxford University Press.

2. Friedlaender JS (editor) (1987) The Solomon Islands Project: A Long-term Study of Health, Human Biology, and Culture Change. New York: Oxford University Press.

3. Hodge AM, Dowse GK, Toelupe P et al. (1997) The association of modernization with dyslipidemia and changes in lipid levels in the Polynesian population of Western Samoa. Int J Epidemiol 26, 297-306.

4. Friedlaender JS (1990) The Solomon Islands Project: an introduction. Am J Phys Anthropol 81, 459-464.

5. Ezeamama AE, Viali S, Tuitele J et al. (2006) The influence of socioeconomic factors on cardiovascular disease risk factors in the context of economic development in the Samoan archipelago. Soc Sci Med 63, 2533-2545.

6. Gelanis DJ, McGarvey ST, Quested C et al. (1999) Dietary intake of modernizing Samoans: implications for risk of cardiovascular disease. J Am Diet Assoc 92, 184-190.

7. Hanna JM \& Fitzgerald MH (1993) Acculturation and symptoms: a comparative study of reported health symptoms in three Samoan communities. Soc Sci Med $\mathbf{3 6}$, 1169-1180

8. Fitspatrick-Nietschmann (1983) Pacific Islanders - migration and health. West J Med 139, 848-853.

9. Hawley NL \& McGarvey ST (2015) Obesity and diabetes in Pacific Islanders: the current burden and the need for urgent action. Curr Diab Rep 15, 29.

10. Mavoa HM \& McCabe M (2008) Sociocultural factors relating to Tongans' and Indigenous Fijians' patterns of eating, physical activity and body size. Asia Pac J Clin Nutr 17, 375-384.

11. McLennan AK \& Ulijaszek SJ (2015) Obesity emergence in the Pacific Islands: why understanding colonial history and social change is important. Public Health Nutr 18, 1499-1505.

12. Widmer A (2013) Diversity as valued and troubled: social identities and demographic categories. Anthropol Med 20, 142-159.

13. Dancause KN, Dehuff C, Soloway LE et al. (2011) Behavioral changes associated with economic development in the South Pacific: health transition in Vanuatu. Am J Hum Biol 23, 366-376.

14. Dancause KN, Vilar M, Wilson M et al. (2013) Behavioral risk factors for obesity during health transition in Vanuatu, South Pacific. Obesity (Silver Spring) 21, E98-E104.

15. Lordan G, Soto EJ, Brown RP et al. (2012) Socioeconomic status and health outcomes in a developing country. Health Econ 21, 178-186.

16. Wang D, Hawley NL, Thompson AA et al. (2017) Dietary patterns are associated with metabolic outcomes among adult Samoans in a cross-sectional study. J Nutr 147, 628-635.
17. Abdi H \& Valentin D (2007) Multiple correspondence analysis. In: Encyclopedia of Measurement and Statistics, pp. 652-657 [N Salkind, editor]. Thousand Oaks, CA: SAGE Publications, Inc.

18. Alaba O \& Chola L (2014). Socioeconomic inequalities in adult obesity prevalence in South Africa: a decomposition analysis. Int $J$ Environ Res Public Health 11, 3387-3406.

19. da Silva VS, Souza I, Silva DAS et al. (2015) Correspondence between overweight and socioeconomic and demographic indicators in the adult Brazilian population. Rev Bras Epidemiol 18, 476-489.

20. Ayele D, Zewotir T \& Mwambi H (2014) Multiple correspondence analysis as a tool for analysis of large health surveys in African settings. Afr Health Sci 14, 1036-1045.

21. Engels T, Baglione Q, Audibert M et al. (2014) Socioeconomic status and stroke prevalence in Morocco: results from the Rabat-Casablanca study. PLoS One 9, e89271.

22. Gesuita R, Skrami E, Bonfanti R et al. (2017) The role of socio-economic and clinical factors of HbA1c in children and adolescents with type 1 diabetes: an Italian multicentre survey. Pediatr Diabetes 18, 241-248.

23. Higuera-Mendieta DR, Cortés-Corrales S, Quintero J et al. (2016) KAP surveys and dengue control in Colombia: disentangling the effect of sociodemographic factors using multiple correspondence analysis. PLoS Negl Trop Dis 10, e0005016.

24. Knoema's World Data Atlas (2017) Vanuatu male obesity prevalence, 1960-2017. https://knoema.com/atlas/ Vanuatu/Male-obesity-prevalence (accessed December 2018).

25. Knoema's World Data Atlas (2017) Vanuatu female obesity prevalence, 1960-2017. https://knoema.com/atlas/ Vanuatu/Female-obesity-prevalence (accessed December 2018).

26. Olszowy KM, Pomer A, Dancause KN et al. (2015) Impact of modernization on adult body composition on five islands of varying economic development in Vanuatu. Am J Hum Biol 27, 832-844.

27. Vanuatu National Statistics Office (2010) 2009 National Population and Housing Census. Volume 1: Basic Tables Report. https://vnso.gov.vu/index.php/component/advlisting/ ?view=download\&fileId=1996 (accessed December 2018)

28. Dancause KN, Vilar M, Chan C et al. (2012) Patterns of childhood and adolescent overweight and obesity during health transition in Vanuatu. Public Health Nutr 15, 158-166.

29. Vanuatu National Statistics Office (2017) 2016 Post-TC Pam Mini-Census Report. Volume 1: Basic Tables. https://vnso.gov. $\mathrm{vu} /$ index.php/component/advlisting/?view $=$ download\&fileId $=$ 4542 (accessed December 2018).

30. Lohman TG, Roche AF \& Martorell R (1988) Anthropometric Standardization Reference Manual. Champaign, IL: Human Kinetics Books.

31. Greenacre M \& Blasius J (editors) (2006) Multiple Correspondence Analysis and Related Methods. Boca-Raton, FL: Chapman \& Hall/CRC Press.

32. Lê S, Josse J \& Husson F (2008) FactoMineR: an R package for multivariate analysis. J Stat Softw 25, 1-18.

33. McDowell MA, Fryar CD, Ogden CL et al. (2008) Anthropometric reference data for children and adults: United States, 2003-2006. Natl Health Stat Report issue 10, 1-48.

34. Gallagher D, Heymsfield SB, Heo M et al. (2000) Healthy percentage body fat ranges: an approach for developing guidelines based on body mass index. Am J Clin Nutr $\mathbf{7 2}$, 694-701.

35. Thow AM (2009) Trade liberalization and the nutrition transition. Mapping the pathways for public health nutritionists. Public Health Nutr 12, 2150-2158. 
36. Hughes R \& Lawrence M (2005) Globalization, food and health in Pacific Island countries. Asia Pac J Clin Nutr 14, 298-306.

37. Sahal Estimé M, Lutz B \& Strobel F (2014) Trade as a structural driver of dietary risk factors for noncommunicable diseases in the Pacific: an analysis of household income and expenditure survey data. Global Health 10, 48.

38. Snowdon W \& Thow AM (2013) Trade policy and obesity prevention: challenges and innovation in the Pacific Islands. Obes Rev 14, Suppl. 2, 150-158.

39. Snowdon W, Raj A, Reeve E et al. (2013) Processed foods available in the Pacific Islands. Global Health 9, 53.

40. Observatory of Economic Complexity (2015) What does Vanuatu import? (1995-2015) http://atlas.media.mit.edu/ en/visualize/line/hs92/import/vut/all/show/1995.2015/ (accessed June 2018).

41. World Bank, World Integrated Trade Solution (2016) Vanuatu country profile. http://wits.worldbank.org/Country Profile/en/VUT (accessed June 2018).

42. DiBello JR, McGarvey ST, Kraft P et al. (2009) Dietary patterns are associated with metabolic syndrome in adult Samoans. J Nutr 139, 1933-1943.
43. Vengiau G, Umezaki M, Phuanukoonnon S et al. (2012) Diet and physical activity among migrant Bougainvilleans in Port Moresby, Papua New Guinea: association with anthropometric measures and blood pressure. Am J Hum Biol 24, 716-718.

44. Republic of Vanuatu, Ministry of Health (2015) Vanuatu Non Communicable disease Policy and Strategic Plan: 20162020. https://www.iccp-portal.org/system/files/plans/ VUT_B3_Vanuatu\%20NCD\%20Policy\%20and\%20Strategic\% 20Plan\%202016-2020.pdf (accessed December 2018).

45. Ives M (2017) As obesity rises, remote Pacific Islands plan to abandon junk food. New York Times, 19 February 2017. https://www.nytimes.com/2017/02/19/world/asia/junkfood-ban-vanuatu.html (accessed December 2018).

46. Weitz CA (2015) The effects of acculturation and age on the exercise capacities of Solomon Islanders. Am J Phys Anthropol 81, 513-525.

47. Martyn T, Yi D \& Fiti L (2015) Identifying the household factors, and food items, most important to nutrition in Vanuatu. http://www.fao.org/fileadmin/user_upload/sap/ docs/Household\%20nutrition\%20analysis\%20Vanuatu\% 202015.pdf (accessed December 2018). 